\title{
A Survey Paper on Internet of Things based Healthcare System
}

\author{
Ms. Shinde Sayali P. ${ }^{1}$, Ms. Phalle Vaibhavi N. ${ }^{2}$ \\ Assistant Professor, Computer Science and Engg Department, AGTI's DACOE, Karad, India ${ }^{1}$ \\ Student, Computer Science and Engg Department, AGTI’s DACOE, Karad, India ${ }^{2}$
}

\begin{abstract}
IOT is the advanced network infrastructure of connectivity, transportation and technology. IOT smart devices can implement the facilities of remote health monitoring and also emergency notification system .IOT has appreciable application of smart healthcare system. In the healthcare system the highlighted policies and strategies that help to the researchers and scientists and experts who develop smart device which is the up-gradation to the existing technology. This survey paper states that how IOT interrelate to various system including the smart healthcare which is one of the prevalent system. Healthcare system has the surveillance that proposed the need of smart devices and smart objects to decrease the inefficiency of available healthcare system. The IOT based healthcare has enhanced technology which is exclusive from the traditional healthcare and whole medical system.
\end{abstract}

Keywords: Internet of things (IOT), surveillance, smart devices, smart healthcare.

\section{INTRODUCTION}

This paper includes the webbed health care and that has the complete base of internet of things .Kelvin Ashton's proposed Internet of things is a concept reflecting a connected any set of anyone, anything, anytime, anyplace, any service, and any network. IOT services network correspondence for system devices and service that override the margins of machine to machine mechanism. IOT allows the object to be sensed to control that creating opportunity for direct integration of physical world into computer based system performance, meticulous and economic benefits.

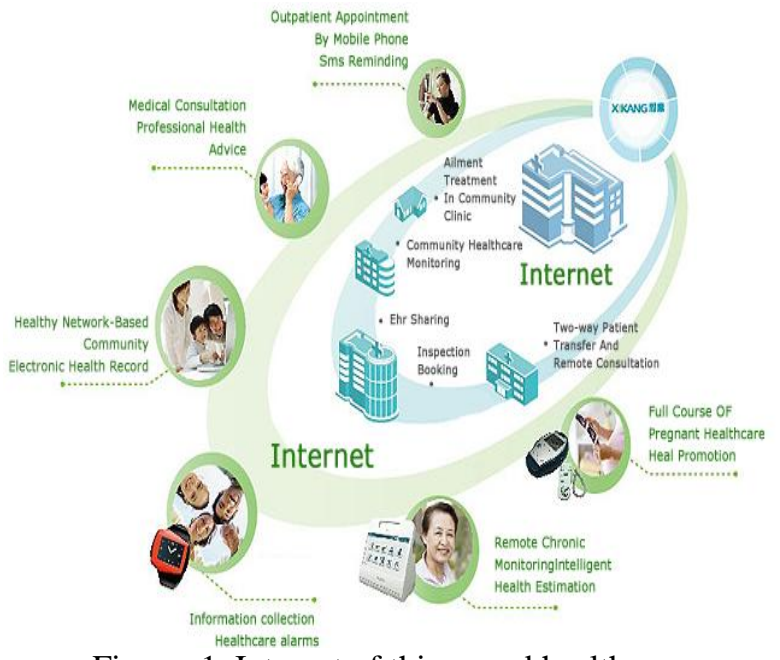

Figure 1. Internet of things and healthcare

\section{RELATED WORK}

- Intelligence wallet for an individual to store big signal and wallet shares.[6]
- For elderly or chronic patients some sensors have developed for human activity monitoring. The role of this system is the continuous monitoring of physiological parameters. [5][1]

- Health care monitors system is mostly depend on wireless sensor network that's why it gives advantage of reduced energy consumption and extend the communication coverage.[4]

- Developing countries have to face the problems like less innovated technology, least availability of smart devices and smart object which is the prevalent need of smart healthcare.[7]

- Developing devices like heart monitoring devices using a wireless sensors and smart phones. It detects the threatening arrhymains when it reaches at the certain threshold value its alarm alerts to patient.[2]

- Applying IOT for personalised health care in smart homes gives service and technology of layered approach.[6]

- Approach of IOT is an IOT aware architecture for smart health system using sensors like temperature sensors, barometric pressure, and ECG sensor. It gives facility like remote monitoring and management of emergency situations [8].

\section{IOT- HEALTHCARE}

Smart servers fix time and passing datato the smart backbone. In current existing health care system due to lack of awareness, poor facility with undeveloped technologies .it the need of the smart health care system . On network hospital help patients and doctors for remote handling of services. Using smart phone applications related to health care system patients may get guidance to 
IARJSET

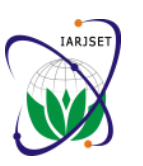

International Advanced Research Journal in Science, Engineering and Technology

National Conference on Innovative Applications and Research in Computer Science and Engineering (NCIARCSE-2017)

AGTI's Dr. Daulatrao Aher College Engineering, Vidyanagar Extension, Karad

Vol. 4, Special Issue 4, January 2017

take decisions suggested by the application. IOT allows to Surveillance has the common outcome those developing tag any patient and able to get health care information by countries have such as the INDIA has Quality assessment the address or database corresponding to particular RFID perspective Population level consideration problems. [4]

(Radio frequency identification).RFID automatically identifies and tracks the tag attached to objects by using electromagnetic fields.[1]The health care mainly issues on diagnosis treatment, health professionals and policies It mainly contained of three layers as follows: added to that the medicinal concerns and public health. The particular health system refers to organisation of people institutions and resources to deliver health care service to meet the need of smart health care the smart health care system based upon IOT includes ehealth and smart devices as tool of up-gradation and future smart healthcare technologies too.[5]

\section{HEALTHCARE SURVEILLANCE}

The smart health care system encounters the smart health disease surveillance. Categorised of this surveillance are mainly smart IOT devices and smart backbone devices. This conclusively serves the mechanisms of cloud computing and main servers at the hospitals. Personal Survey and an international response followed by overall public health by World Health Organization (WHO) that has announced plans to establish the Disease Intelligence Unit that will function independently. The trends and analysis have reached the backbone network that is the prevalent need of today. Smart diseases surveillance is extreme novel and innovation to speed up the existing process of surveillance to reach the highest goals of accuracy and real-time database. Smart disease surveillance is an epidemiological practice by which outbreak and spreading can be monitored. Main role is to predict and to observe to minimise harm to the lives. Modern communication technology includes organisation like world health organisation WHO and centres for disease control that now can report with enhanced and advanced application like 'smart grid' and smart devices like heart monitoring ,biochip, transponders, etc.[7]

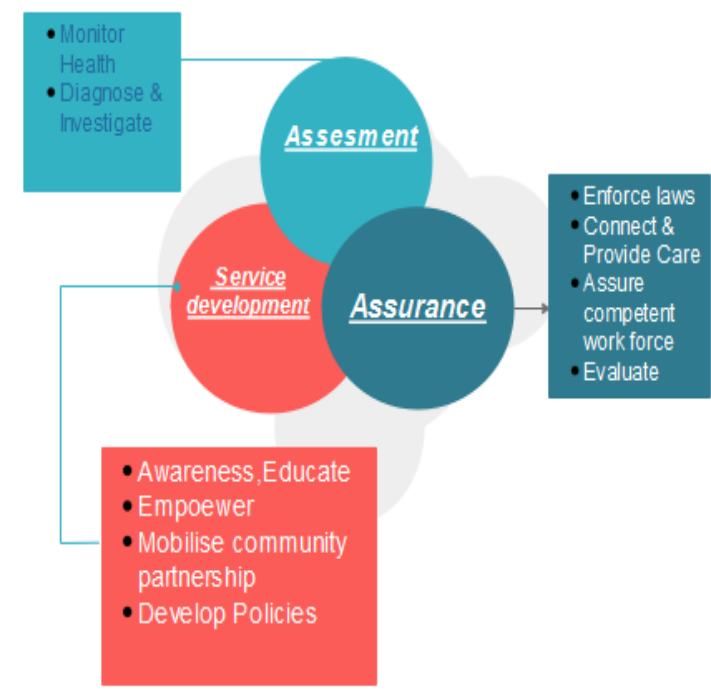

Figure 2.system management in smart healthcare

A. Perception layer

C. Application layer

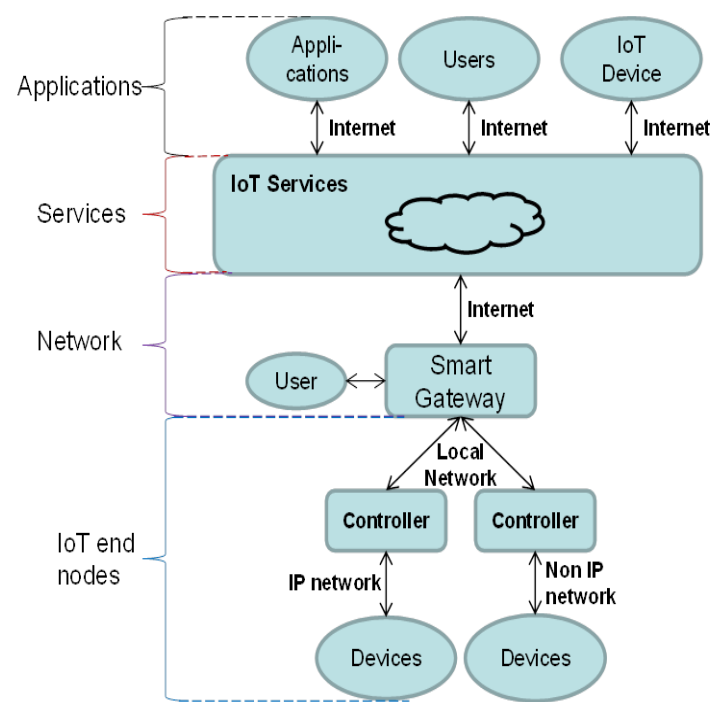

Figure 3. Architecture of IOT based healthcare

\section{LOGICAL STRUCTURE}

To develop a logical structure to access the information from portable platform built on medical personnel and administrative personnel. Every user can allow accessingof this integrated information depending upon its score roll through this platform.[6][2]

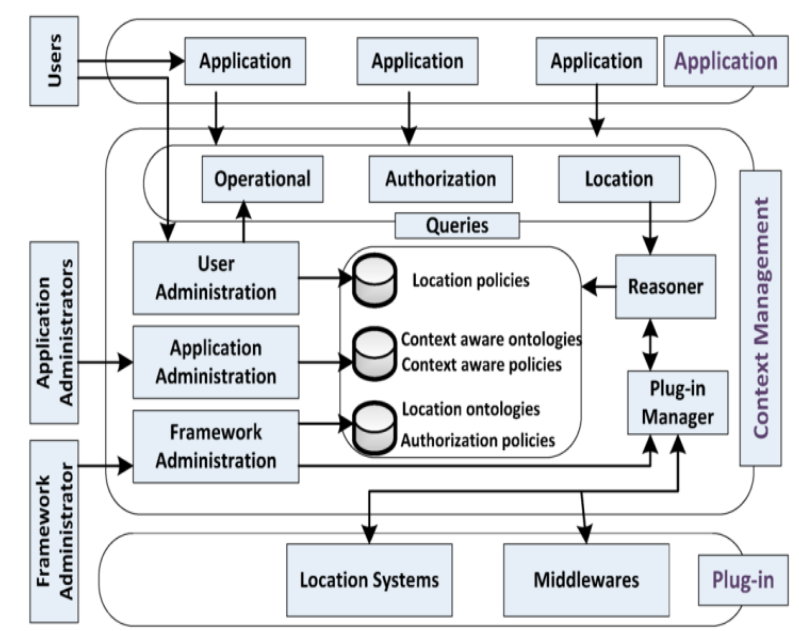

Figure 3.Logical structure of smart healthcare

\section{V.SMART DEVICES}

IOT is increasingly being recognised by researchers and analyst as one of the most sophisticate technology that has 
IARJSET

the potential of innovation. It consists of sensors, actuators, computing device and data communication capabilities. It enhances advance treatments and diagnosis that has improved drastically. Smart devices are used to treat the issues like chronic disease management, personal health and fitness management.[3]

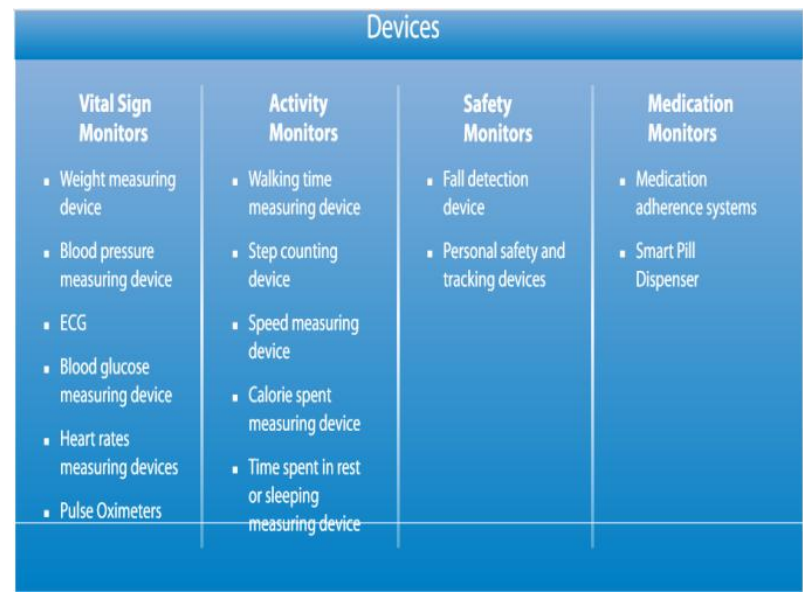

Figure 5. IOT technology - smart medical devices

\section{INTERNET OF THINGS - MEDICAL DEVICES}

$>$ Access real-time visibility about overall patient condition.

$>$ Address the immerging challenges in smart healthcare

$>$ Managing device diversity and interoperability (like peripheral area network).

> Integrating data that has understood intelligent application like correlation complex event Processing and automated reasoning with sematic technology.

> Maintaining efficiency, flexibility and evolutionary applications by analysing typical 'Big data' problem, velocity of data ingestion alsostringent real time performance and dedicated purpose built-up apps.

$>$ Serves medical master lines or proficiency by providing features like 'Data securitisation, Scale volume data, Patient-device Interaction, etc.[5]

\section{CONCLUSION}

- Smart health care system has enhanced features due to IOT. Intelligence of work has been acquiring with the help of premises of IOT.

- Health care system has minimized complication and complexity withthe environment of IOT.

- All the predictions that we got from the survey of whole medical system are un-prosperous maintenance and unutilized technology.

- This problem isovercome by full-fledged utilization of upgraded and new generation technology.

- Evolving the smart health care device is feasible way to manipulate existing healthcare.

- Intensification of awareness of smart diseases and implementation of government schemes to improve quality of life.

\section{ACKNOWLEDGMENT}

We would like to give the special thanks to the computer science engineering department of the college DACOE HOD Prof. Ashish Patil to have their guidance. We are also thankful to the technologies that we have used to have such format of paper.

\section{REFERENCES}

[1]. S. M. RIAZUL ISLAM1, (Member, IEEE), DAEHAN KWAK2, MD. HUMAUN KABIR1, MAHMUD HOSSAIN3, AND KYUNG-SUP KWAK1, (Member, IEEE) 1UWB Wireless Communications Research Center, Inha University, Incheon 402751, Korea Networked Systems and Security Laboratory, Rutgers University, Newark, NJ 07102, USA 3Secure and Trustworthy Computing Laboratory, University of Alabama at Birmingham, AL 35233, USA "The Internet of Things for Health Care: A Comprehensive Survey", 1 june 2015

[2]. Ms. Supriya Chandrakant Padwal M.E. (VLSI \& ES), Dept. of E\&TC Sahyadri Valley College of Engg. \& Tech. Pune, India. Mr. Suraj Vishnu Kurde Department of Computer Engg. Sahyadri Valley College of Engg. \& Tech. Pune, India "Long-Term Environment Monitoring for IOT Applications using Wireless Sensor Network", February 2016.

[3]. Steve Warren, Ph.D. and Richard L. Craft, M.S. Sandia National LaboratoriesJohn T. Bosma Potomac Institute for Policy Studies "Designing Smart Health Care Technology into the Home of the Future",25 march 1999.

[4]. Rashmi Singh "A Proposal for Mobile E-Care Health Service System Using IOT for Indian Scenario", 1 January 2016

[5]. Ashok Khanna Business Head UK, EU and Major Accounts, Life Sciences (EIS) Global Head, Presales and Solutions for Life Sciences (EIS ) Prateep Misra Research Area Manager, TCS Innovation Labs "The Internet of Things for Medical Devices Prospects, Challenges and the Way Forward".

[6]. Lei Yu School of Computer and Information, Hefei University of Technology, Hefei, China 2 School of Medical Information Technology, Anhui University of Traditional Chinese Medicine, Hefei, China Email: fishstonehfut1006@163.com Yang Lu, XiaoJuan Zhu School of Computer and Information, Hefei University of Technology, Hefei, China Email: luyang.hf@126.com,xjzhu@aust.edu.cn "Smart Hospital based on Internet of Things", 10 October 2012.

[7]. Ashly Mathew1, Farha Amreen S.A2, Pooja H.N3, Aakriti Verma4 "Smart Disease Surveillance Based on Internet of Things (IoT)"

[8]. Sreekanth K U PG Scholar Department of Computer Science \& Engineering Vidya Academy of Science \& Technology (VAST) Thrissur, India sreekanthku1@gmail.com ,Nitha K P Asst. Professor Department of Computer Science \& Engineering"A Study on Health Care in Internet of Things"

\section{BIOGRAPHIES}

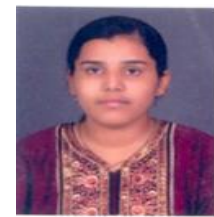

Ms. Sayali P. Shinde, Assistant Professor, At AGTI's DACOE, Karad, M.E At Pune University, Area of interest Data mining, information retrieval, security.

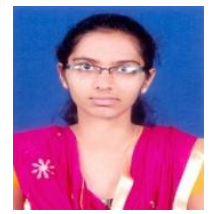

Ms. Vaibhavi N.Phalle, Student, CSE Department, At AGTI's DACOE. 